\title{
Target-oriented Time-lapse Waveform Inversion using Virtual Survey
}

Di Yang*, Yingcai Zheng, Michael Fehler, and Alison Malcolm, MIT

\section{SUMMARY}

Time-lapse seismic data are widely used for monitoring timevariant subsurface changes. Conventional analysis provides qualitative information by comparing results from consecutive surveys, whereas waveform inversion can retrieve quantitative estimates of reservoir properties through seismic waveform fitting. The quantitative evaluation of the physical parameters obtained by waveform inversion allows for better interpretation of fluid substitution and migration during processes like oil and gas production, and carbon sequestration. Since reservoir changes are localized and only part of the data are of interest, the time-lapse waveform inversion can be optimized in terms of computational cost and convergence rate. In this study, we propose a scheme of localized waveform inversion with computed datasets we refer to as virtual surveys. Both the model domain and trace duration in forward modeling are reduced by the reorganization of the data. We show a numerical example in which the recovery of the reservoir change is computationally faster and more robust to source-receiver locations than inversion with original survey.

\section{INTRODUCTION}

Time-lapse seismic monitoring is often applied in reservoir management in the oil industry to obtain information about reservoir changes. It helps identify bypassed oil to be targeted for infill drilling, and extends the economic life of a field (Lumley, 2001). It is also capable of monitoring the progress of fluid fronts providing information for injection optimization in enhanced oil recovery and long-term fluid storage like carbon sequestration (Bickle et al., 2007). Generally, one baseline survey and subsequent monitoring surveys are acquired over time. Analysis and comparison of the datasets provides an estimate of changes in seismic velocity and mass density. These changes are related to the changes in dynamic reservoir properties like pore pressure and fluid saturation (Dadashpour et al., 2008) that are important in reservoir simulation and interpretation. To quantitatively recover these physical parameters, full waveform inversion (FWI) (Tarantola, 1984; Virieux and Operto, 2009) is beginning to be tested on individual surveys. The application of FWI to time-lapse data seems straight-forward, however, the high nonlinearity and costly computation motivate further research on inversion strategies tailored for timelapse data.

Although FWI is becoming more feasible with increasing computing power, it is still time-consuming because of the large computational domain and large number of required iterations due to the slow convergence of the method. Since we are only interested in the time-variant changes in the reservoir, reducing the large portion of the computation that would be devoted to evaluating structures outside the reservoir will expedite the process and allow more focus to be placed on the reservoir it- self. Moreover, inversions for the entire model domain might induce a poor recovery of volumetric information within the reservoir from surface reflection acquisitions. The high nonlinearity might cause spurious images when directly subtracting independent inversion results (Yang et al., 2011). A localized inversion focused on the reservoir with only the corresponding data has the potential to overcome these issues.

In this work, we present a target-oriented waveform inversion scheme specialized for time-lapse reservoir monitoring. Baseline surface survey data are used in a standard waveform inversion to obtain a baseline model. The signal changes between the baseline and time-lapse datasets sensitive to the reservoir changes are transformed to a new datum at the top of the reservoir. New data sets are synthesized from the recorded data with the re-datumed signals and the new virtual survey geometry. A time-lapse waveform inversion is conducted in a reduced model domain to extract the information about the reservoir changes. We describe the virtual survey technique first, and then use synthetic examples to show the advantage of our method.

\section{THEORY}

Seismic redatuming is essential in most imaging techniques for exploration purposes. Various methodologies have been proposed to project the surface survey data into the subsurface to form constructive images based on the concept of survey sinking (Claerbout, 1985). The datasets are recursively synthesized at increasing depth by applying an appropriate phase shift to the data:

$$
P_{z}(\omega, R, S)=e^{i k_{z} z} *^{(R)} e^{i k_{z} z}{ }^{(S)} P_{z-\delta z}(\omega, R, S)
$$

where $P_{z}(\omega, R, S)$ and $P_{z-\delta z}(\omega, R, S)$ are the wavefields at depth $z$ and $z-\delta z$ respectively. The first convolution operation $(*(S))$ downward continues the receiver gathers, and the second convolution operation $\left(*^{(R)}\right)$ downward continues the source gathers.

However, the phase shift correction is not enough for waveform inversion as it does not accurately account for amplitude changes. Assuming that we have obtained a reasonable baseline model from the standard waveform inversion that fits the baseline survey, we can calculate the Green's function between any two points using finite difference forward modeling. Only the shallower part of the model that is above the reservoir (between the actual survey line and the virtual survey line) is involved in this calculation. We can calculate the Green's function $G_{i, j}$ from each virtual receiver $V R_{i}$ to each surface receiver $S R_{j}$. The signal recorded by the $S R_{j}$ can be written as:

$$
P_{S R_{j}}(\omega)=P_{V R_{i}}(\omega) * G_{i, j}(\omega)
$$

Standard backpropagation will approximate the inverse of the 


\section{waveform inversion with virtual survey}

matrix $G_{i, j}$ using its adjoint $G^{*}{ }_{j, i}$ :

$$
P_{V R_{i}}(\omega) \approx P_{S R_{j}}(\omega) * G^{*}{ }_{j, i}(\omega)
$$

This effectively reduces the amplitude of the waves by applying the Green's function twice.

Since we can calculate the Green's function explicitly, the $P_{V R_{i}}(\omega)$ can be derived by:

$$
P_{V R_{i}}(\omega)=P_{S R_{j}}(\omega) * G^{*}{ }_{j, i}(\omega) *\left(G_{i, j}(\omega) * G^{*}{ }_{j, i}(\omega)\right)^{-1}
$$

The matrix $G_{i, j}(\omega) * G^{*}{ }_{j, i}(\omega)$ is positive definite, and for a practical number of receivers, the computation of the inverse of the matrix is not difficult. The term $\left(G_{i, j}(\omega) * G^{*}{ }_{j, i}(\omega)\right)^{-1}$ serves as an amplitude correction during the data backpropagation. (a)

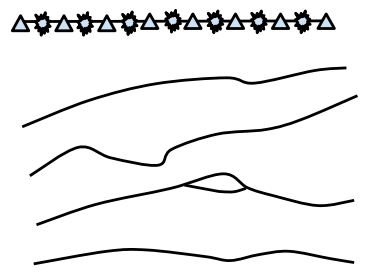

(c)

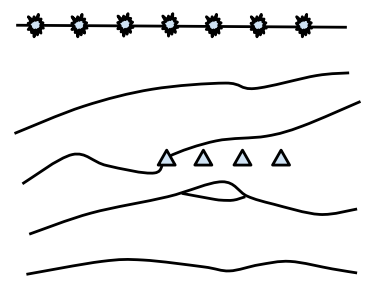

(e)

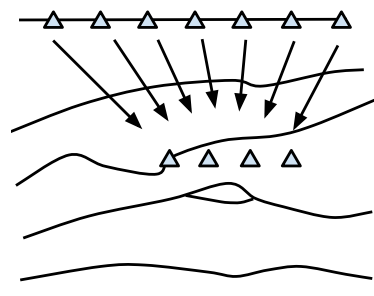

(b)

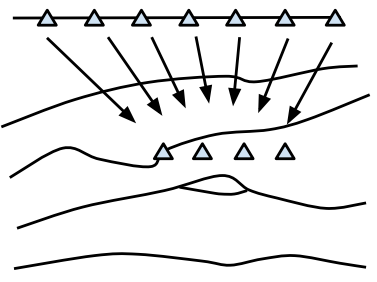

(d)

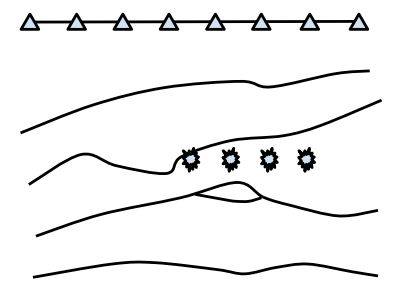

(f)

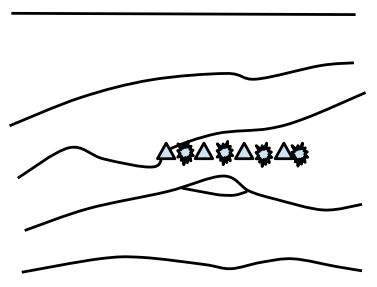

Figure 1: (a) The original survey on the surface with a lot of sources and receivers. (b) The receiver field on the surface is re-datumed into the subsurface for each shot. (c) Now the survey consists of sources on the surface and receivers in the subsurface. (d) By applying reciprocity, the survey equivalently consists of sources in the subsurface and receivers on the surface. (e) The new receiver field in (d) is re-datumed into the subsurface. (f) The virtual survey is formed with sources and receivers in the subsurface after the process above.

After re-datuming the surface shot gathers $P_{S R_{j}}$, we have the shot gathers at the virtual receiver locations $P_{V R_{i}}$ (Figure 1(c)). With reciprocity, we consider these virtual receivers as sources (virtual sources $V S_{i}$ ) and the surface sources as receivers and obtain virtual source gathers $P_{V S_{i}}$ at the surface (Figure 1(d)).
With the process described above, the wave records $P_{V S_{i}}$ are propagated from the surface to the virtual receiver depth (Figure 1(e)) to form a "virtual survey" which is now independent of the upper part of the model (Figure 1(f)). The whole process not only reduces the model size, but also makes use of the data redundancy to reduce the size of the data domain. There are fewer sources and receivers in the virtual survey than in the actual survey, and only the data that focus on the reservoir are kept in the virtual survey.

This survey sinking style process described above is very similar to the idea of extended images (Sava and Vasconcelos, 2011). An extended image can be defined by evaluating the scattered field for finite source-receiver offsets and at nonzero times. In our study, the virtual survey data are essentially the scattered field from the subsurface below the virtual survey depth evaluated for finite source-receiver offsets.

With the synthesized datasets, we can conduct a full waveform inversion within a much smaller model domain. The objective function to be minimized is

$$
E(m)=\frac{1}{2}\left|u_{\text {virtual }}-u_{\text {modeling }}\right|^{2}=\frac{1}{2} \delta u^{T} \delta u
$$

where $u_{\text {virtual }}$ and $u_{\text {modeling }}$ are the synthesized virtual survey data and forward modeling data with the virtual sourcereceiver geometry based on the baseline model, respectively, $\delta u=u_{\text {virtual }}-u_{\text {modeling }}$, the superscript ${ }^{T}$ denotes the transpose, and $m$ is the parameter (velocity and density) to be updated. Based on the assumption that the change from the baseline model is relatively small, the objective function can be minimized via a gradient-based method.

The gradient of the objective function is derived by taking its derivative with respect to $m$, leading to

$$
\nabla_{m} E=\frac{\partial E(m)}{\partial m}=\left(\frac{\partial u_{\text {modeling }}}{\partial m}\right)^{T} \delta u
$$

This can be calculated efficiently by cross-correlating the forward propagating wavefields from sources with the back propagating the residual wavefield from receivers (Tarantola, 1984). We use the nonlinear conjugate gradient method to update the model in each iteration, which has both low computational cost and fast convergence. The inversion process is discussed in more details in (Yang et al., 2011).

\section{SYNTHETIC EXAMPLE}

We use a layered acoustic model as shown in Figure 2 to demonstrate the power of the method. In the time-lapse model shown in Figure 3, the model has a reduction in P-wave velocity ($200 \mathrm{~meters} / \mathrm{s}$ ) in the reservoir location as indicated. $350 \mathrm{re}-$ ceivers and 200 sources are used on the surface to generate the datasets. Figure 4 shows one shot gather for each dataset. The source is a $25 \mathrm{~Hz}$ Ricker wavelet. In this synthetic study, we assume the baseline model is known. With the knowledge of the approximate location of the reservoir, we can use the part of the model that is above the reservoir to calculate Green's functions used to estimate data for the virtual survey. We compare the performance of a standard time-lapse FWI with one 


\section{waveform inversion with virtual survey}

with the virtual survey to show its advantages in speed and convergence.

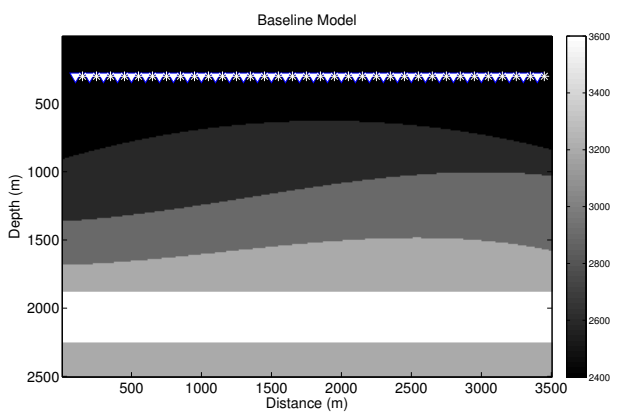

Figure 2: The baseline model and the surface survey geometry.

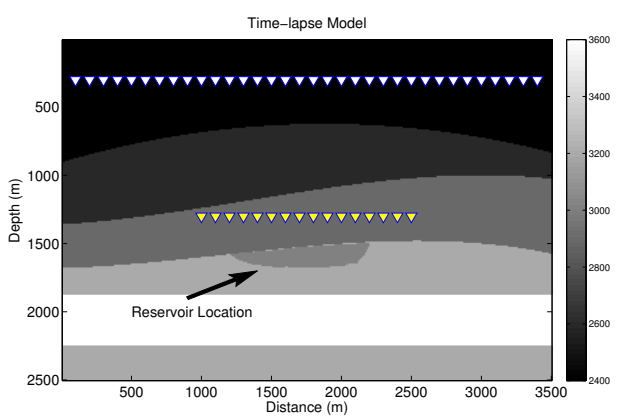

Figure 3: The time-lapse model and the virtual survey geometry (yellow triangles).

\section{VIRTUAL SURVEY}

The only data that can be correctly projected to the virtual survey are those reflected from below the virtual survey depth. We only project the data difference that is shown in Figure 4(c). At the new survey depth, 5 receivers are used to record the projected data from each shot. After reciprocity, these receivers became shots in the receiver gathers. We project the receiver gathers and recorded the field with 200 receivers. We use numerical simulation to generate virtual baseline datasets with the 5 virtual sources and 200 virtual receivers in the baseline model. The virtual time-lapse datasets are additions of virtual baseline datasets and the virtual receiver gathers obtained above as shown in Figure 5.

\section{INVERSION RESULTS COMPARISON}

The standard waveform inversion was performed for both the original time-lapse data and the virtual survey data. Five sources are used in both methods. The 5 virtual sources are computed from all 200 surface sources as described above. Figure 6 shows the inverted model change at iteration 1,10 and 30 for both methods. In both cases, the shape of the velocity anomaly
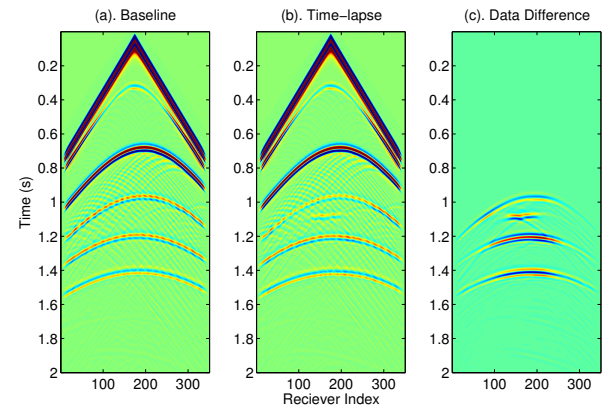

Figure 4: (a). one baseline shot gather of a surface survey; (b). one time-lapse shot gather of a surface survey; (c). data difference between the two datasets
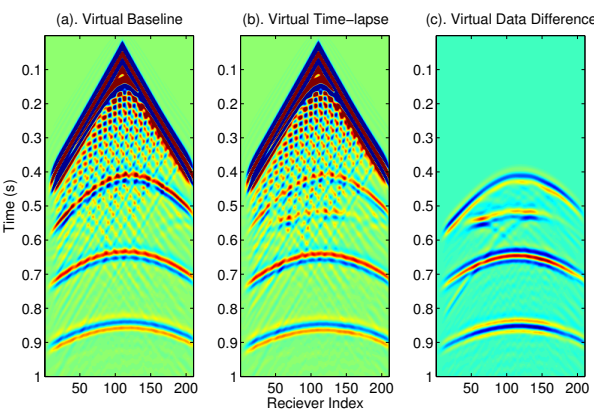

Figure 5: (a). Synthetic baseline survey with virtual geometry; (b). Synthesized time-lapse survey with synthetic baseline survey and projected data difference;(c). Projected data difference

is well recovered from the reflection signals from the first iteration, similar to the results of reverse time migration. But the amplitude inside the volume is lower than the true value. At each iteration shown in Figure 6, the recovery of the velocity amplitude with the virtual survey method is always better than the standard method. The convergence of the inversion with the virtual survey addressed through the cost function minimization is also faster as shown in Figure 7.

\section{DISCUSSION}

From a computational point of view, the virtual survey method expedites the calculation by reducing the model size and record duration. For standard waveform inversion, to obtain a reasonable recovery for the reservoir, 20 to 30 iterations are needed. Each iteration involves $N_{s} * N_{l s}$ times forward modelings, where $N_{s}$ is the number of sources, and $N_{l s}$ is the number of iterations used for each line search. In our $2 \mathrm{D}$ synthetic example, the model domain is reduced to $40 \%$ of the original size. And the duration is half of the original time. Each forward modeling only takes $20 \%$ of the original calculation. And to achieve the same model recovery, fewer iterations are needed. In 3-D, the saving would be more significant.

To use all the sources in a standard waveform inversion is also 

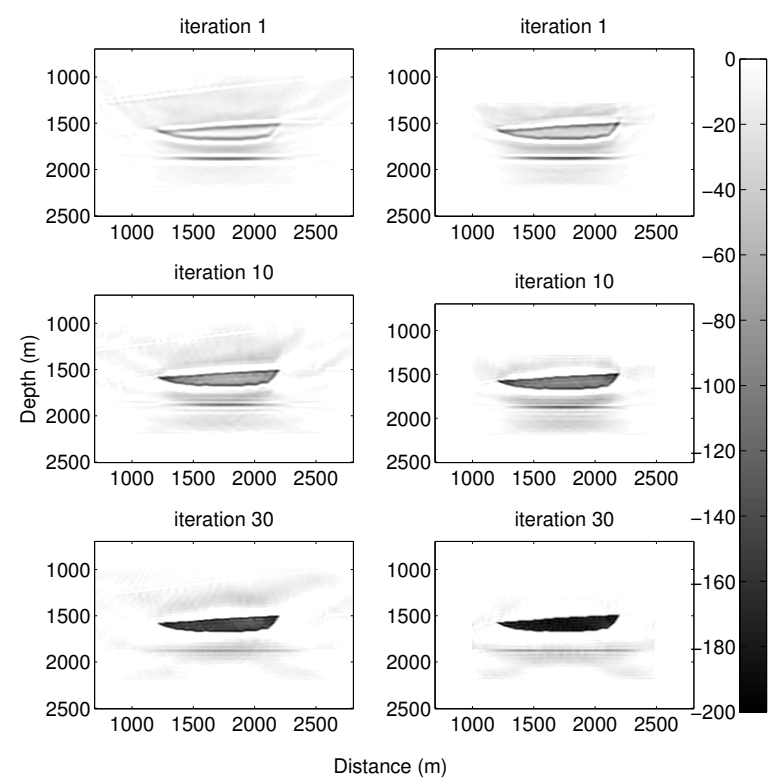

Figure 6: The comparison between inversion results with a surface survey and virtual survey. The inversion with virtual survey recovers the velocity better in each iteration showed in the figure.

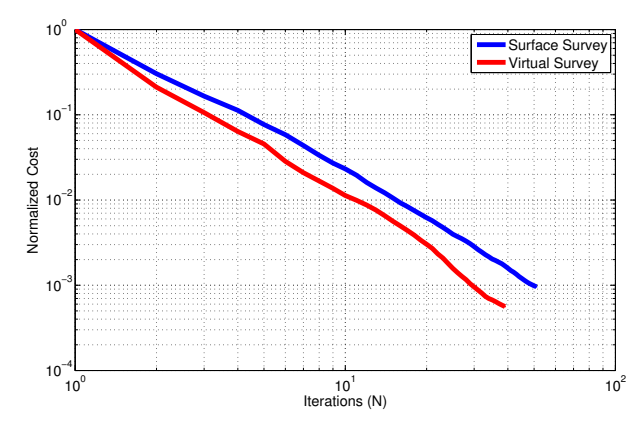

Figure 7: Normalized cost function reductions over iterations for surface survey and virtual survey are compared. The virtual survey inversion converges faster.

costly. Phase encoding (Krebs et al., 2009) was proposed to reduce the computation but also introduces cross-talk. In our method, the data from different shot gathers are synthesized together to form the virtual data. If the same number of sources for the two methods are used, our method makes use of more information from the original surveys.

The virtual survey itself needs Green's function calculations between virtual receivers and actual receivers, and between sources and virtual sources. It takes $N_{v r}+N_{v s}$ times forward modelings within the upper part of the model, which are highly parallelizable.

Another advantage of this method is that the virtual surveys can be synthesized at the same source-receiver locations for both baseline and time-lapse data, even if the actual surveys have different geometries. This allows the application of doubledifference waveform inversion (Denli and Huang, 2009) which improves the time-lapse image quality by reducing the contamination from local minima.

The convergence slows down as the iteration number increases for both the standard inversion and the one with the virtual survey. The full recovery of the volumetric change (the value inside the anomaly) is always difficult. One reason for this is that the forward scattering signals (waves transmitted through the anomaly) are weaker than the backscattering signals. It is possible to make use of the virtual survey idea to enhance the forward scattering signals as shown in Figure 8. The virtual receivers below the reservoir utilize large-offset signals which might be omitted by the virtual receivers above the reservoir during back-propagation since the virtual survey has a much smaller offset range. The new survey geometry will have both surface survey and partial cross-well survey. The cost function is reweighed to expedite the recovery for the volumetric change. However, to calculate the wavefield below the reservoir, we need to know the velocity model below the reservoir. And the ray-path of these large-offset waves from the actual receiver to the virtual receiver as indicated in Figure 8 should not pass through the reservoir.

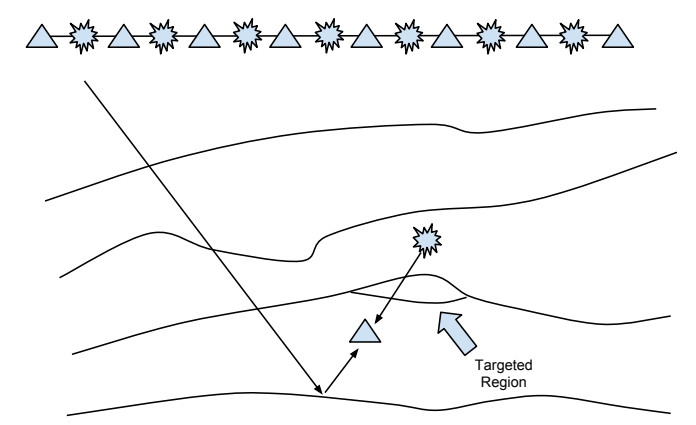

Figure 8: Transform the reflection into transmission.

\section{CONCLUSIONS}

We have proposed an optimized target-oriented time-lapse waveform inversion method. The original surface survey is transformed into a new survey geometry that focuses on the reservoir region. The synthetic example shows that our method is computationally more efficient, and converges faster compared to a standard waveform inversion. More importantly, the recovery of the volumetric change inside the reservoir is closer to the true value.

\section{ACKNOWLEDGMENTS}

This work was supported by the MIT Earth Resource Laboratory Founding Members Consortium. 
http://dx.doi.org/10.1190/segam2012-1308.1

\section{EDITED REFERENCES}

Note: This reference list is a copy-edited version of the reference list submitted by the author. Reference lists for the 2012 SEG Technical Program Expanded Abstracts have been copy edited so that references provided with the online metadata for each paper will achieve a high degree of linking to cited sources that appear on the Web.

\section{REFERENCES}

Bickle, M., A. Chadwick, H. Huppert, M. Hallworth, and S. Lyle, 2007, Mode ling carbon dioxide accumulation at Sleipner: Implications for underground carbon storage: Earth and Planetary Science Letters, 255, 164-176.

Claerbout, J., 1985, Imaging the earth's interior: Blackwell Science Inc.

Dadashpour, M., M. Landrø, and J. Kleppe, 2008, Nonlinear inversion for estimating reservoir parameters from time-lapse seismic data: Journal of Geophysics and Engineering, 5, 54.

Denli, H., and L. Huang, 2009, Double-difference elastic waveform tomography in the time domain: $79^{\text {th }}$ Annual International Meeting, SEG, Expanded Abstracts, 28, 2302-2306.

Krebs, J., J. Anderson, D. Hinkley, R. Neelamani, S. Lee, A. Baumstein, and M. Lacasse, 2009, Fast fullwavefield seismic inversion using encoded sources: Geophysics, 74, no. 6, WCC177-WCC188.

Lumley, D., 2001, Time-lapse seismic reservoir monitoring: Geophysics, 66, 50-53.

Sava, P., and I. Vasconcelos, 2011, Extended imaging conditions for wave-equation migration: Geophysical Prospecting, 59, 35-55.

Tarantola, A., 1984, Inversion of seismic reflection data in the acoustic approximation: Geophysics, 49, 1259-1266.

Virieux, J., and S. Operto, 2009, An overview of full-waveform inversion in exploration geophysics: Geophysics, 74, no. 6, WCC1-WCC26.

Yang, D., M. Fehler, A. Malcolm, and L. Huang, 2011, Carbon sequestration monitoring with acoustic double-difference waveform inversion: A case study on SACROC walkaway VSP data: 82nd Annual International Meeting, SEG, Expanded Abstracts, 30, 4273-4277. 